\title{
When Malignancies Mimic Interstitial Lung Disease: A Case Series and Review of the Literature
}

\section{Guler $\mathbf{S}^{1, *}$, Berezowska $\mathbf{S}^{2}$, Christe $\mathbf{A}^{3}$, Funke $\mathbf{M}^{1}$ and Geiser $\mathbf{T}^{1}$}

${ }^{1}$ Department of Pulmonary Medicine, University Hospital and University of Bern, Switzerland

${ }^{2}$ Institute of Pathology, University of Bern, Switzerland

${ }^{3}$ Department of Diagnostic, Interventional and Pediatric Radiology, University Hospital and University of Bern, Switzerland

\begin{abstract}
Interstitial lung diseases (ILD) are difficult to diagnose and to classify. Despite symptoms and clinical findings typical for ILD, a broad differential diagnosis must be kept in mind. Multidisciplinary discussion (MDD) contributes to correct diagnosis and is therefore considered the diagnostic gold standard in ILD. Here we report on three patients that were referred to our ILD clinic with suspected ILD. They all presented with chronic dyspnea on exertion, cough and abnormal lung function tests showing reduced diffusing capacity as well as hypoxemia. Chest CT scans were compatible with ILD. Further diagnostic workup was performed, revealing two adenocarcinomas of the lung and one malignant B cell lymphoma. In summary, despite clinical, physiological and radiological presentation typical for ILD, further workup is needed early in the course of the disease to rule out possible differential diagnoses including malignancies.
\end{abstract}

Keywords: Interstitial lung disease; Multidisciplinary discussion; Lung cancer; Adenocarcinoma; B cell lymphoma

\section{Introduction}

Interstitial lung diseases (ILD) represent a variety of disorders that affect the lung parenchyma and cause unspecific respiratory symptoms such as increasing dyspnea and cough. Correct diagnosis and classification of ILD is crucial in order to avoid exposures that may be responsible for ILD, to decide about specific treatment with novel antifibrotic drugs $[1,2]$ and to inform the patient about prognosis of the disease. Classification of ILD may be challenging with a spectrum from ILD with known etiologies (occupational and environmental exposures, drugs or connective tissue diseases) to idiopathic forms (idiopathic interstitial pneumonias, e.g. idiopathic pulmonary fibrosis [IPF]). Based on the current guidelines [3,4], a histological diagnosis for IPF is not always mandatory and depends on the clinical and radiological presentation. Bronchoscopy and bronchoalveolar lavage (BAL) are not routinely recommended.

We present three cases that were referred to our ILD clinic with respiratory symptoms, physiological and radiological findings typical for ILD. Further workup was performed, resulting in a different diagnosis than initially suspected.

\section{Case Reports}

\section{Case 1}

A 65 year old Caucasian male and current smoker (40 pack years) was referred to the ILD clinic with increasing dyspnea at minimal physical efforts. The retired locksmith stated an exposure to hard metal dust by tool grinding over decades. Arterial hypertension was treated with candesartan and depression with citalopram. Physical examination showed an overweight (BMI $29 \mathrm{~kg} / \mathrm{m}^{2}$ ), non-febrile patient, with bilateral, basal, inspiratory crackles, without clinical signs of heart failure. Laboratory blood tests (CRP, hematology, liver and renal functions) were normal, but mild respiratory insufficiency was detected in arterial blood gas analysis $\left(\mathrm{pO}_{2} 66 \mathrm{mmHg}, \mathrm{pCO}_{2} 49\right.$ $\mathrm{mmHg}$ ). Pulmonary function tests (PFT) showed severe obstructive airflow limitation (FVC 2.14l/49\% predicted, FEV1 0.72l/21\% predicted) and moderately decreased adjusted diffusing capacity for carbon monoxide (DLCO) of $42 \%$ predicted. Chest CT scan showed an interstitial disease pattern with subpleural reticulations, disseminated, multiple small parenchymal nodules and slightly enlarged mediastinal and hilar lymph nodes (Figure 1a). Initial differential diagnosis included sarcoidosis and hard metal lung. Further diagnostic workup with bronchoscopy including BAL and transbronchial biopsy (TBB), revealed adenocarcinoma of the lung with lymphangitis carcinomatosa, positivity for thyroid transcription factor (TTF)-1 (Figure 1b), without epidermal growth factor receptor (EGFR) mutations or anaplastic lymphoma kinase (ALK) rearrangements. Contralateral pulmonary and spinal metastases were detected on CT scan. A palliative chemotherapy with carboplatin and permetrexed was started, to which the patient responded well.

\section{Case 2}

A 76 year old Caucasian female, never-smoker and housewife, was referred for the evaluation of recurrent cough with whitish sputum and progressive dyspnea on exertion. Except for esomeprazole (treatment of gastroesophageal reflux) the patient was on no regular medication. She presented with velcro rales (discontinuous, inspiratory, non-musical crackles). Laboratory tests showed anemia (hemoglobin $116 \mathrm{~g} / \mathrm{l}$ ), but no signs of inflammation, metabolic disturbances, rheumatologic or autoimmune diseases. The patient was hypoxemic (pO2 $58 \mathrm{mmHg}$ ) and PFT revealed mild restrictive limitation (FVC 1.871/90\% predicted) and a mild decrease in adjusted DLCO (70\% predicted). A chest CT scan showed mainly right sided ground-glass opacities and dense

*Corresponding author: Sabina Guler, MD, Department of Pulmonary Medicine, Inselspital, University Hospital Berne, CH-3010 Bern, Switzerland, Tel: +41-31632-2111; Fax: +41-31-632-1404; E-mail: sabina.guler@insel.ch

Received December 18, 2015; Accepted December 29, 2015; Published December 31, 2015

Citation: Guler S, Berezowska S, Christe A, Funke M, Geiser T (2015) When Malignancies Mimic Interstitial Lung Disease: A Case Series and Review of the Literature. J Clin Respir Dis Care 1: 104. doi: 10.4172/ JCRDC.1000104

Copyright: (c) 2015 Guler S, et al. This is an open-access article distributed under the terms of the Creative Commons Attribution License, which permits unrestricted use, distribution, and reproduction in any medium, provided the original author and source are credited. 


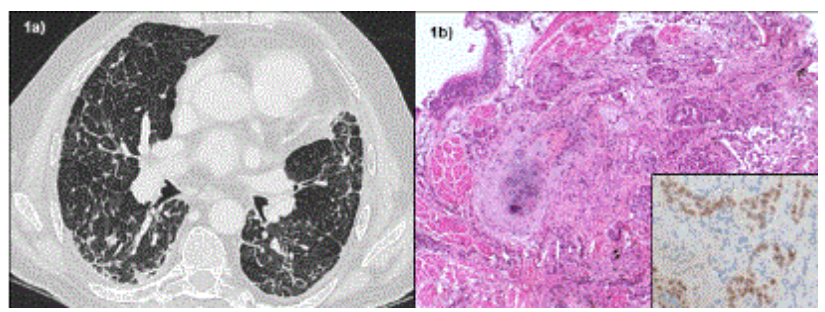

Figure 1: (a) Chest CT scan: Subpleural reticulations, disseminated, multiple small parenchymal nodules, bihilar and mediastinal lymphadenopathy. (b) Transbronchial biopsy: adenocarcinoma of the lung with lymphangiosis carcinomatosa (H\&E, original magnification 100x, inlet: TTF-1).

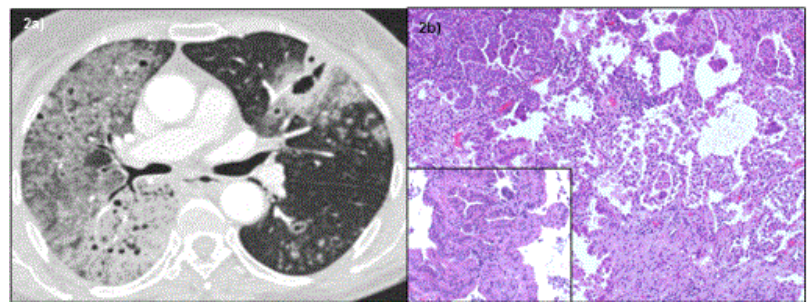

Figure 2: (a) Chest CT scan: Predominantly right-sided GGO, dense consolidations with air bronchogram, cystic changes and tree-in-bud pattern. (b) Surgical lung biopsy: Mulifocal adenocarcinoma with predominantly lepidic growth pattern (H\&E, original magnification 50x, inlet:original magnification 200x)

consolidations with air bronchogram and cystic changes (Figure 2a). Small caverns and tree-in-bud pattern were also present on the left side, and all changes showed a basal predominance. In face of the radiological presentation, the suspected diagnosis was cryptogenic organizing pneumonia. Bronchoscopy, BAL and TBB were not diagnostic. Finally, surgical lung biopsies revealed multifocal adenocarcinoma, cytokeratin (CK)7 positive, TTF-1 negative, with a predominantly lepidic growth pattern without EGFR mutation or ALK rearrangement (Figure $2 b$ ). No evidence of distant metastases or primary tumors as possible origins of the pulmonary malignancy were found by further staging. The patient equally received palliative chemotherapy with carboplatin and permetrexed, but died 6 months later after 6 cycles of chemotherapy.

\section{Case 3}

A 59 year old asian female, never smoker, was referred for suspected ILD of unknown origin. She reported dry cough, wheezing and dyspnea on physical effort during the last 4 months. The patient had received empiric treatment with oral steroids for her suspected ILD during the last 2 months prior to referral, which ameliorated her symptoms to some extent. She presented with bilateral, inspiratory crackles, hypoxemia (pO2 $65 \mathrm{mmHg}$ ), severe anemia (hemoglobin $73 \mathrm{~g} / \mathrm{l}$ ), and a severe restrictive limitation (FVC $0.791 / 37 \%$ predicted) on PFT. CT scan of the lungs showed multiple, bilateral noduli, consolidations and groundglass opacities with bihilar and mediastinal lymphadenopathy (Figure 3a). Drug-induced ILD was suspected since she was on metformin due to diabetes [5]. BAL revealed $47 \%$ lymphocytes with $35 \%$ atypical B cells on flow cytometry. Biopsy of a left, supraclavicular lymph node finally showed a low-grade malignant follicular B cell lymphoma WHO grade II (Figure 3b), with bone marrow involvement in the staging biopsy. A modified COP chemotherapy (cyclophosphamide, vincristine, prednisone) including rituximab was administered.

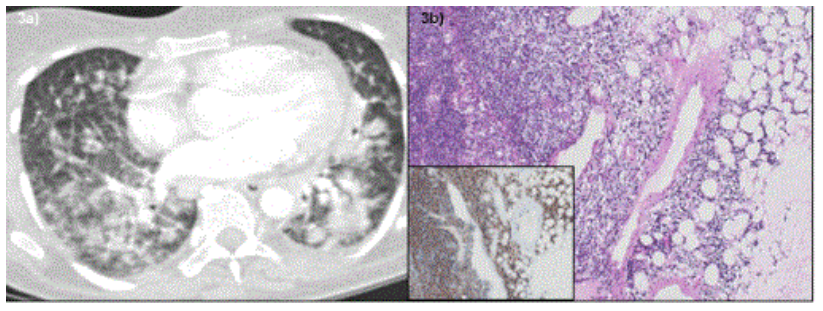

Figure 3: (a) Chest CT scan: Multiple bilateral noduli, consolidations, GGO bihilar and mediastinal lymphadenopathy. (b) Histology of the supraclavicular lymph node: Follicular B cell lymphoma (H\&E, original magnification 100x, inlet: CD20).

\section{Discussion}

We present three cases referred to our ILD clinic with suspected ILD based on clinical and radiological presentation. Further workup, however, revealed malignant disease. Since the clinical presentations of ILD patients are often unspecific, high-resolution CT scans of the chest are performed. Despite correlation with the clinical picture during MDD this does not always result in a clear diagnosis. As the reported three cases illustrate, a broad differential diagnosis needs to be considered, including malignant disease.

Our cases speak in favour of acquiring histological diagnosis, in particular if the clinical and radiological presentations are unclear or not conclusive. On the other hand especially in elderly patients with comorbidities, the necessity of invasive procedures should be carefully accessed and the diagnostic intervention carefully planned, keeping in mind possible complications of invasive procedures, such as surgical lung biopsy, with bronchoscopy being less invasive.

Adenocarcinoma of the lung has been reported in the literature mimicking "benign" processes such as pneumonia [6], desquamative interstitial pneumonia (DIP) [7] or lung fibrosis with honeycombing [8]. Lymphangiosis carcinomatosa can also present as ILD due to thickening of the bronchovascular bundles prominent in the axial compartment and/or thickened peripheral interlobular septa shaping a polygonal pattern [9]. The typical fibrotic changes in reaction to the proliferation of malignant cells (desmoplastic reaction) can further contribute to misdiagnosis. Lymphoma, another differential diagnosis of ILD with malignant etiology, may show lymphangitic spread with the radio-pathological changes mentioned above [10].

Although BAL is not recommended in the current ATS/ERS recommendations for ILD on a regular basis [3], we routinely perform BAL in patients with suspected ILD, primarily to rule out infections [11] or malignancies. Poletti et al. [12,13] report a diagnostic yield of $77 \%$ for adenocarcinoma, $93 \%$ for bronchioloalveolar carcinoma (adenocarcinoma in situ and adenocarcinoma with predominant lepidic growth pattern) and 67\% for Non-Hodgkin lymphoma.

In the three reported cases, malignancies presented as ILD. But ILD may also co-exist with malignant disease. In these cases, the diagnosis of a neoplasm is particularly important and challenging due to several overlaps. Chronic inflammation leads to impaired epithelial cell reparation, with proliferation and metaplasia [14] and cytologically atypical epithelial proliferations can be demonstrated in areas of honeycombing. On the other hand, a recent review $[15,16]$ recorded an explicitly higher prevalence of lung cancer in the IPF population of $4.8 \%-48 \%$ versus $2 \%-6.4 \%$ in the non-IPF affected cohort. The prognosis of IPF is worse in those patients, making lung cancer an 
Citation: Guler S, Berezowska S, Christe A, Funke M, Geiser T(2015) When Malignancies Mimic Interstitial Lung Disease: A Case Series and Review of the Literature. J Clin Respir Dis Care 1: 104. doi: 10.4172/ JCRDC.1000104

important adverse factor concerning morbidity and mortality [17]. Lung cancer treatment and the development of ILD are also associated: the development of fibrotic lung parenchymal changes after radio- and chemotherapy has been repeatedly demonstrated [18]. In addition, cancer treatment is postulated to cause acute exacerbation of ILD and may trigger a "latent" ILD [19]. The question if malignant tumors themselves can trigger the development of extensive lung fibrosis has not yet been resolved.

In summary, these case series and findings support our standard practice to perform bronchoscopy and BAL and, if rendered necessary after MDD, surgical lung biopsies, in patients with suspected ILD. Only in cases of matching clinical findings and UIP pattern on CT scans IPF can be diagnosed without tissue diagnosis by experienced ILD specialists $[3,20]$. Similarly as in cancer care, decision-making about diagnostic steps, final diagnosis and treatment in ILD benefits from the opinion of several experts, especially pulmonologists, radiologists and pathologists. Therefore MDD should be integrated in clinical ILD routine like multidisciplinary tumor boards are part of the oncologic routine.

\section{References}

1. King TE, Bradford WZ, Castro-Bernardini S, Fagan EA, Glaspole I, et al. (2014) A phase 3 trial of pirfenidone in patients with idiopathic pulmonary fibrosis. $\mathrm{N}$ Engl J Med 370: 2083-2092.

2. Richeldi L, du Bois RM, Raghu G, Azuma A, Brown KK, et al. (2014) Efficacy and safety of nintedanib in idiopathic pulmonary fibrosis. N Engl J Med 370: 2071-2082.

3. Travis WD, Costabel U, Hansell DM, King TE, Lynch DA, et al. (2013) An official American Thoracic Society/European Respiratory Society statement: Update of the international multidisciplinary classification of the idiopathic interstitial pneumonias. American journal of respiratory and critical care medicine 188 733-748.

4. Demedts M, Costabel U (2002) ATS/ERS international multidisciplinary consensus classification of the idiopathic interstitial pneumonias. Eur Respir J 19: 794-796.

5. www.pneumotox.com.

6. Thompson WH (2004) Bronchioloalveolar carcinoma masquerading as pneumonia. Respiratory care 49: 1349-1353.

7. Raparia K, Ketterer J, Dalurzo ML, Chang YH, Colby TV, et al. (2014) Lung tumors masquerading as desquamative interstitial pneumonia (DIP): report of 7 cases and review of the literature. Am J Surg Pathol 38: 921-924.

8. Lantuejoul S, Colby TV, Ferretti GR, Brichon PY, Brambilla C, et al. (2004) Adenocarcinoma of the lung mimicking inflammatory lung disease with honeycombing. Eur Respir J 24: 502-505.

9. Stein MG, Mayo J, Muller N, Aberle DR, Webb WR, et al. (1987) Pulmonary lymphangitic spread of carcinoma: appearance on CT scans. Radiology 162: 371-375.

10. Khojeini EV, Song JY (2014) Intravascular large B-cell lymphoma presenting as interstitial lung disease. Case Rep Pathol 928065(10): 15.

11. Meyer KC, Raghu G, Baughman RP, Brown KK, Costabel U, et al. (2012) An official American Thoracic Society clinical practice guideline: the clinical utility of bronchoalveolar lavage cellular analysis in interstitial lung disease. American journal of respiratory and critical care medicine 185: 1004-1014.

12. Poletti V, Romagna M, Allen KA, Gasponi A, Spiga L (1995) Bronchoalveolar lavage in the diagnosis of disseminated lung tumors. Acta Cytol 39: 472-477.

13. Poletti V, Poletti G, Murer B, Saragoni L, Chilosi M (2007) Bronchoalveolar lavage in malignancy. Semin Respir Crit Care Med 28: 534-545.

14. Konigshoff M (2011) Lung cancer in pulmonary fibrosis: tales of epithelial cell plasticity. Respiration 81: 353-358.

15. Hironaka M, Fukayama M (1999) Pulmonary fibrosis and lung carcinoma: a comparative study of metaplastic epithelia in honeycombed areas of usua interstitial pneumonia with or without lung carcinoma. Pathol Int 49: 1060-1066.

16. Li J, Yang M, Li P, Su Z, Gao P, et al. (2014) Idiopathic pulmonary fibrosis will increase the risk of lung cancer. Chin Med J 127: 3142-3149.

17. Tomassetti S, Gurioli C, Ryu JH, Decker PA, Ravaglia C, et al. (2015) The impact of lung cancer on survival of idiopathic pulmonary fibrosis. Chest 147 : 157-164.

18. Abid SH, Malhotra V, Perry MC (2001) Radiation-induced and chemotherapyinduced pulmonary injury. Curr Opin Oncol 13: 242-248.

19. Yano T, Koga T, Ninomiya S, Taketomi A, Yoshida T, et al. (2002) A review of Japanese literatures concerning surgery for lung cancer with idiopathic interstitial pneumonia. Kyobu Geka 55: 131-133.

20. Raghu G, Collard HR, Egan JJ, Martinez FJ, Behr J, et al. (2011) An officia ATS/ERS/JRS/ALAT statement: idiopathic pulmonary fibrosis: evidence-based guidelines for diagnosis and management. American journal of respiratory and critical care medicine 183: 788-824. 